مدى احتياجات زراع القمح للمعارف التكنولوجية الزراعية الحديثة المتعلقة بتحسين إنتاجية القمح ومواصفاته بمنطقة غرب النوبارية في محافظة البحيرة

$$
\text { أحمد محمد على غزلان }
$$

لتحسين إنتاجية القمح ومواصفاته، حيث بلغت نسبتهم حوالي ץ,qءء٪، فضلاً عن وجود قصور في المعارف

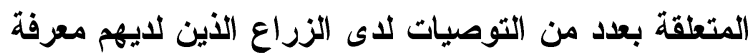

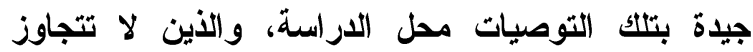

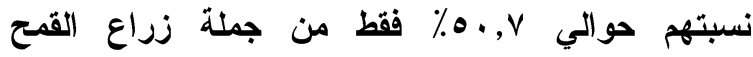

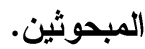

r- تبين من خلال استخدام الارجات الترجيحية القياسية للاحتياج المعرفي، أن أعلى قيمة تعبر عن مستوى الاحتياج

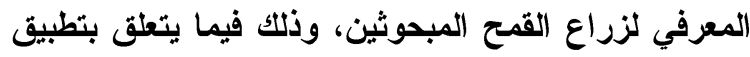

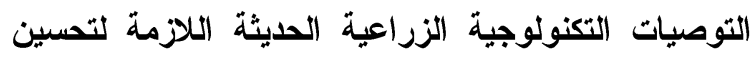
إنتاجية القمح ومواصفاته هي ه ؛ درجة، وأدنى قيمة هي r درجة، وتم توزيع المبحوثين، وفقاً لمستويات احتياجهم المعرفي إلى ثلاث فئات هي: فئة منففضي الاحتياج المعرفي وتبلغ نسبتهم حوالي هـ، وفئة متوسطي الاحتياج

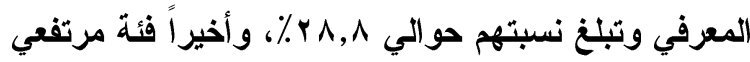

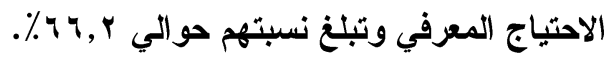
ـ- وفى ضوء نتائج التحليل الإحصائي والكمي، تبين أن أهم المتغيرات ذات التأثير على مستوى الاحتياجات المعرفية للمبحوثين، فيما يتعلق بتطبيق التوصيات التكنولوجية

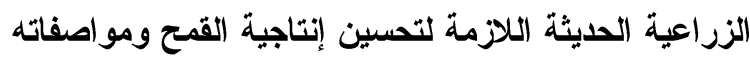
هي: الاستعداد للتغيير وتقبل الأفكار الجديدة، والمساحة المنزرعة قماً، ومتوسط إنتاجية الفدان. ومن ثم يجب أن الن النائ

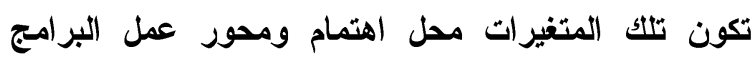

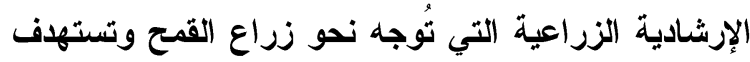

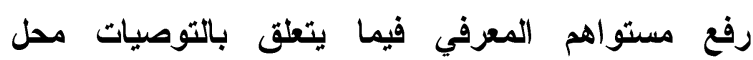
الار اسة.
الملخص العربى

استهذف هذا البحث بصفة رئيسية تحديد مدى احتياجات

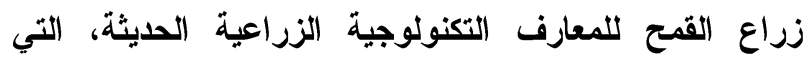

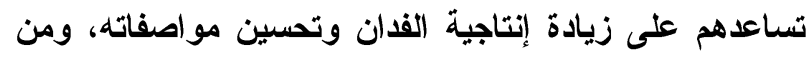
ثم تحديد إلى أي مدى يمكن أن تساعد الأجهزة الإرشادية

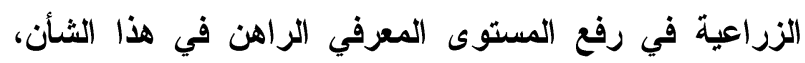

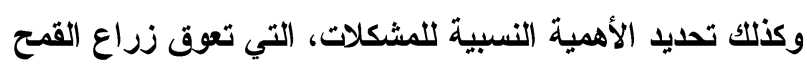
عن زيادة معارفهم المتعلقة بتلك الموضوع. وقد تم جمع الاهيه البيانات اللازمة للاراسة باستخدام الاستبيان، بالمقابلة

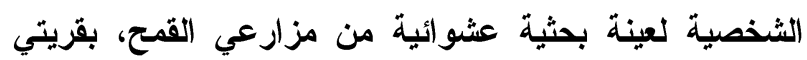
الإمام مالتك، والصديق يوسف بمنطقة غرب النوبارية بمحافظة

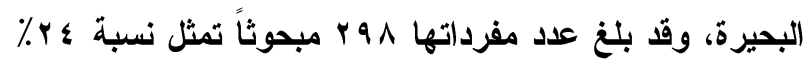
من شاملة البحث.

ومن الدراسة التحليلية الوصفية والإحصائية لمستوى الاحتياجات المعرفية لزراع القمح، فيما يتعلق بتطبيق التوصيات

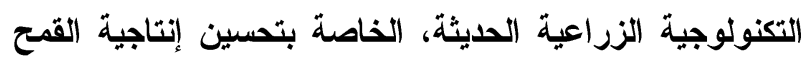

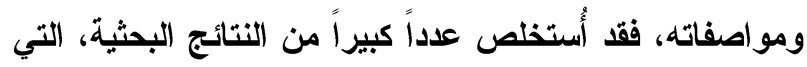
تم الاستناد إليها في التوصيات وأهمها ما يلي: 1 - اتضح من خلال استخدام الدرجات المعيارية الخاصة بقياس المستوى المعرفي، أن غالبية زراع القمح المبحوثين، كانوا

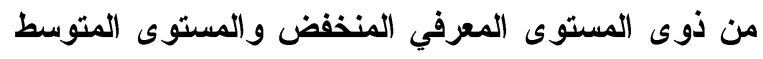

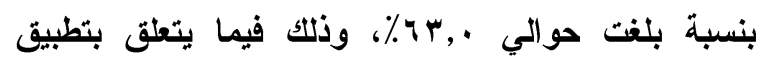

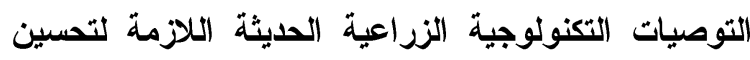
إنتاجية القمح ومواصفاته، ومن ثم شدة احتياجاتهم المعرفية المتعلقة بهذا الثأن.

r- تبين ارتفاع نسبة زراع القمح المبحوثين، الذين لا يعرفون

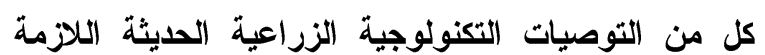

'باحث بمعهد بحوث الإرشاد الزراعي والتتمية الريفية مركز البحوث الزر اعية

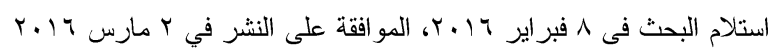


1- تحديد المستوى المعرفي لزراع القمح المبحوثين، و إلى الى

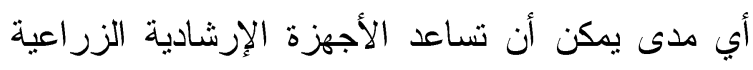
في رفع المستوى المعرفي الر اهن في هذا الثأن. r- دراسة أهم العوامل المؤثرة على مستوى الاحتياجات

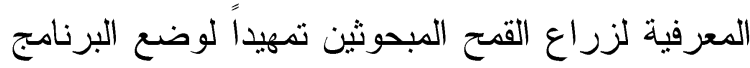

$$
\text { اللازم لتحقيق الهدف الرئيسي. }
$$

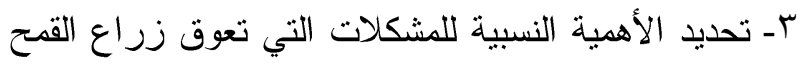
المبحوثين عن زيادة معارفهم المتعلقة بتطبيق التوصيات التكنولوجية الزراعية الحديثة اللازمة لتحسين إنتاجية

$$
\text { القمح ومو اصفاته. }
$$

\section{الإطار النظري والاستعر اض المرجعي}

يعتبر الإرشاد الزراعي نشاطاً تعليمياً يستهدف تنمية

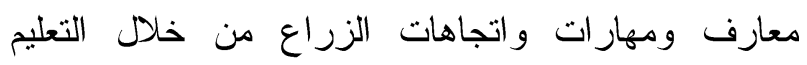

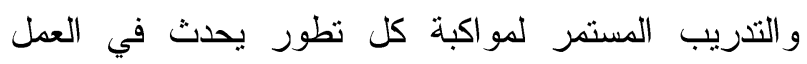

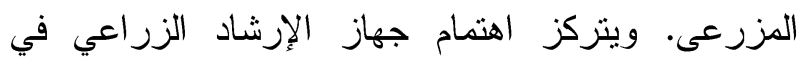

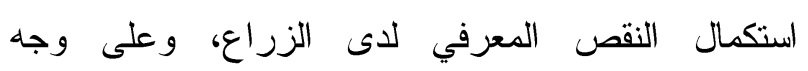

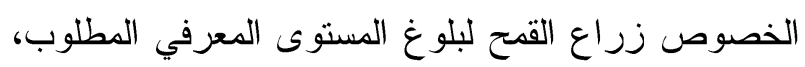
للاستفادة الكاملة من التكنولوجيا الزراعية الحديثة في إنتاج

ولاثك أن النتائج الفنية الزراعية والإرشادية، المستخلصة من الدراسات الإرشادية الزراعية، تساعد لدد كبير على إعداد البرامج الإرشادية اللازمة لتتمية مهارات

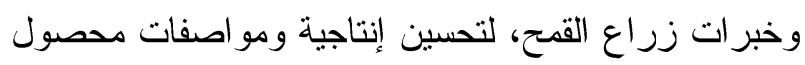
القمح.

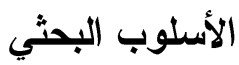

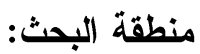

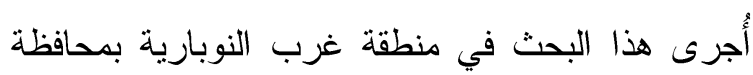

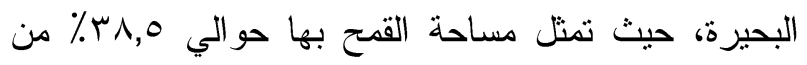
إجمالي المساحة المنزرعة، وقد تم اختبار قريتي الإمام مالك والصديق يوسف التابعتين للنطقة غرب النوبارية
ه- تبين من خلا استخام الارجات الترجيحية القياسية، أن أهم المشكلات التي تعوق زراع القمح المبحوثين عن زيادة

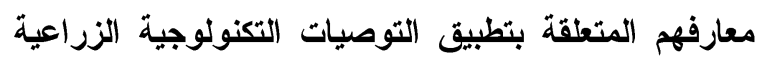
الحديثة اللازمة لتحسين إنتاجية القمح ومواصفاته، وفقاً لإجاباتهم على الترتيب العام التنازلي التالي: انخفاض سعر التهر

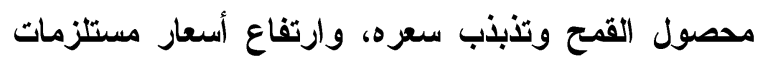

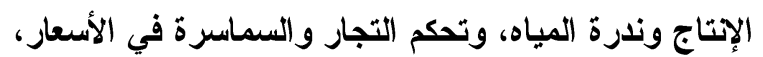
ويُعد الأسواق عن أماكن الإتتاج، وارتفاع أجور العمالة الزراعية وندرتها وقلة إنتاجيتها، وارتفاع تكلفة وعام الانداع

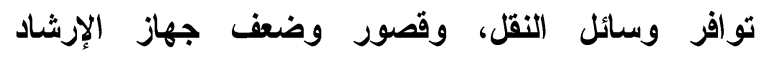
الزراعي، حيث تراوحت الأهمية النسبية للارجات الأنيات

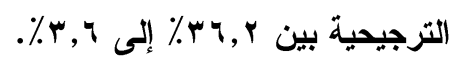

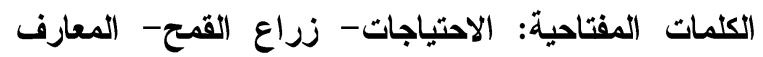
التكنولوجية الزراعية الحيثة- تحسين الإتتاجية والمواصفات

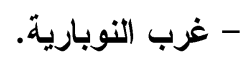

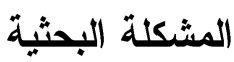

على الرغم من أن مر اكز البحوث المختلفة، تسعى نحو

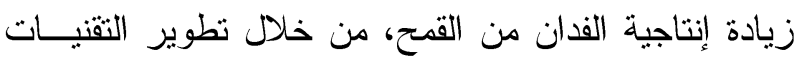

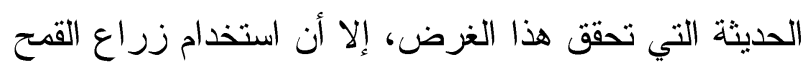

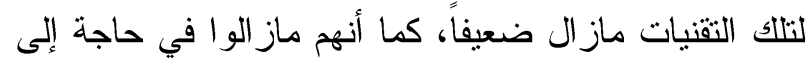

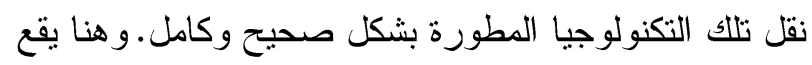
على كاهل مر اكز الإرشاد الزر اعي حل تلك المشكلة، بمـــا

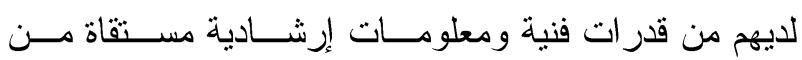

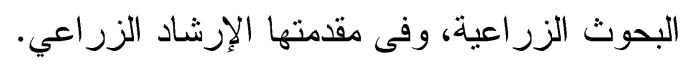

\section{الأهداف البحثية}

يستهف هذا البحث بصفة رئيسية، تحديد هدى احتياجات زراع القمح بمنطقة غرب النوبارية في محافظة البحيرة، إلى المعارف التكنولوجية الزراعية الحديثة، التي تساعدهم على زيادة إنتاجية الفدان وتحسين مواصفاته. ويمكن تحقيق هذا الهدف الرئيسي من خلال دراسة كل من: 
a

= ob

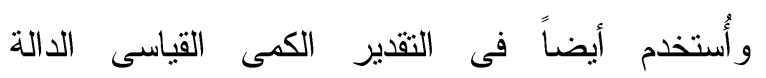
اللو غاريتمية المزدوجة وهى: $\operatorname{Ln} y=\operatorname{Ln} b_{0}+b_{1} \operatorname{Ln} x_{1}+b_{2} \operatorname{Ln} x_{2}+\ldots \ldots+b_{n} \operatorname{Ln} x_{n}$

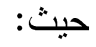

= Ln y التوصيات التكنولوجية الزر اعية الحديثة اللازمة لتحسين

$$
\begin{aligned}
& \text { إنتاجية القمح ومو اصفاته. } \\
& \text { Ln a }
\end{aligned}
$$

Ln x 1 ... Ln xn قيم المرونات الجزئية للمتغيرات المستقلة $=b_{1} \quad \ldots \quad b_{n}$ الداخلة فى الدالة.

\section{أهم المفاهيم الإجرائية البحثية \\ المستوى المعرفي:}

يقصد به درجة إلمام زراع القمح المبحوثين بالتوصيات

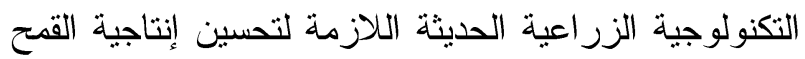
ومواصفاته، ويتم التعبير عنه بقيمة رقمية يمكن التوصل إليها من خلال إجابة المزارع المبحوث على ثلاثة عشر سؤالاً تتعلق بتلاك التوصيات، وقد ثم تقسيمه إلى ثلاثة

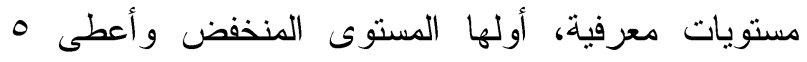
درجات ويقصد بها الدرجة الترجيحية القياسية (المعيارية) التي تعبر عن أهمية المتغير بالنسبة للمتغيرات الأخرى، وثانيها المستوى المتوسط وتم إعطائه · 1 درجات، أما

$$
\begin{aligned}
& \text { المستوى المرتفع فأخذ } 10 \text { درجة. } \\
& \text { الاحتياجات المعرفية: }
\end{aligned}
$$

يُقصد بها مقدار الفجوة بين معارف زراع القمح المبحوثين الحالية وبين المعارف المرغوبة فيما يتعلق بتطبيق التوصيات التكنولوجية الزراعية الحديثة اللازمة لتحسين إنتاجية القمح ومواصفاته، ويتم التعبير عنها بقيمة
لإجر اء الدراسة بهما، نظراً لارتفاع الأهمية النسبية لأعداد

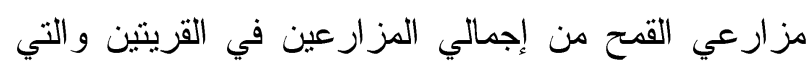

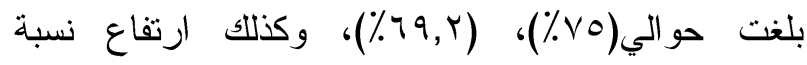
مساحات القمح المنزرعة والتي بلغت حوالي(^,9.7\%)،

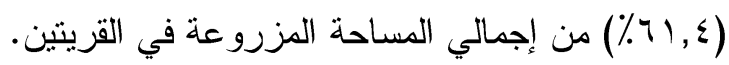
الثاملة و العينة البحثية:

تمثلت شاملة هذا البحث في جميع مزارعي القمح بقريتي الإمام ماللك و الصديق يوسف التابعتين لمنطقة غرب هربع

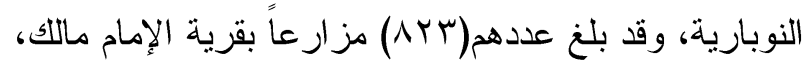
مزارعاً بقرية الصديق يوسف. وتم سحب عينة (1) ) عشوائية ممثلة للشاملة من خلال كشوف حصر مزارعي

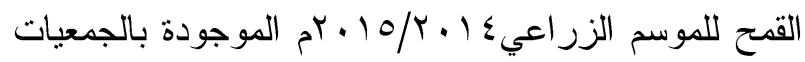

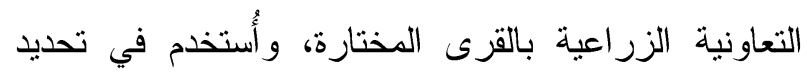
عدد مفردات العينة معادلة كريجسى ومورجان عند مستوى بلري

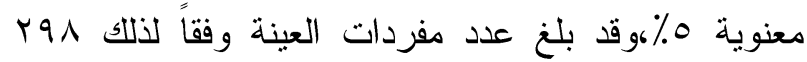

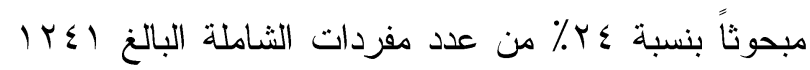

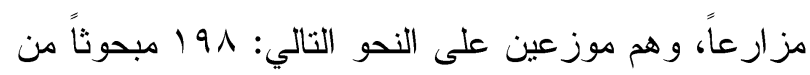
قرية الإمام مالك، . ․ . مبحوثاً من قرية الصديق يوسف.

\section{المنهج التحليلي:}

تم إجراء التحليل الوصفي و التحليل الإحصائي و الكمي، حيث أُستخدم الانحدار المتعدد(Multi-Regression Analysis)

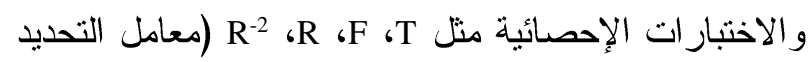
المعدل) للتأكد من صحة النتائج المتحصل عليها من الناحية الإحصائية وذلك عند مستوى معنوية (0\%). وقد أُستخدم في من من عن التقدير الكمي القياسي الدالة الخطية أو الدرجة الأولى $y=a \pm b x$
حيث:

= المتغير المستقل الواحد (One Independent Variable). 
زماعة محصول القمح بعد ب-ـ أيام من الحقن مع (V) عدم التأخير في الزر اعة لعدم فقد الأمونيا. الإستعداد للتغيير وتقبل الأفكار الجديدة: تم توزيعه إلى ثلاثة مستويات، حيث أعطى للمستوى المنخفض r درجة ويقصد بها الدرجة الترجيحية القياسية (المعيارية) التي تعبر عن أهمية المتغير بالنسبة للمتغيرات الأخرى، وأعطى للمستوى المنوسط ؛ درجات، ونم إعطاء

$$
\text { المستوى المرتفع } 7 \text { درجات. }
$$

تم تقسيمها إلى ثلاث فئات، أقلها في المساحة(؟ ب- ؟ ج)

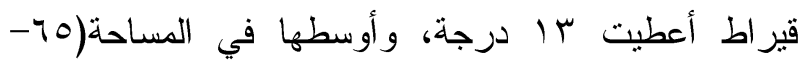
ع • () قير اط تم إعطائها جr درجة، و أكبرها في المساحة

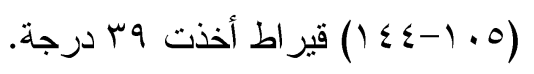

متوسط إنتاجية (لفدان:

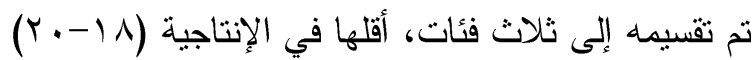

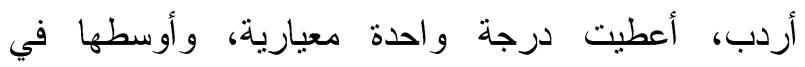

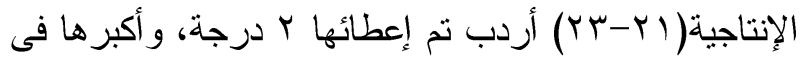

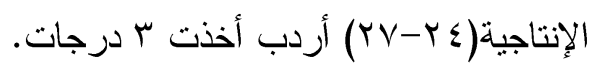

\section{النتائج البحثية و المناقثة}

أولاً: المستوى المعرفي لاى زراع القـح المبحوثين: أوضحت نتائج الدراسة أن الددى الفعلي للقيم الرقمية المعبرة عن المستوى المعرفي للمبحوثين قد تزاوح بين حد

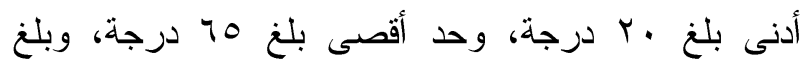
المتوسط الحسابي العام لقيمة المستوى المعرفي بالنسبة للعينة البحثية rك درجة. ولقد تم تصنيف زراع القمح المبحوثين وفقاً لمستو اهم المعرفي محل الاهتمام من خلال استخدام الدرجات المعيارية إلى ثلاث فئات، الفئة المنخفضة

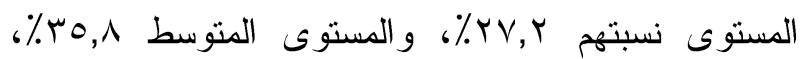
و المستوى المرتفع • ,Vr\% من المجموع الكلى للمبحوثين
رقمية يمكن التوصل إليها في ضوء النظرة المعيارية للحاجة، وذلك بطرح القيمة الرقمية المشاهدة (الفعلية) التي حصل عليها المبحوث من إجابته على جميع الأسئلة الخاصة بتحديد مستواه المعرفي الواقعي من الحد الأقصى

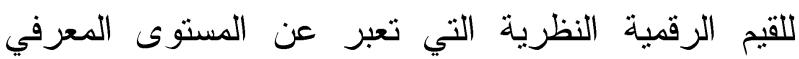
المر اد تحقيقه، و التي يمكن أن يحصل عليها المبحوث عندما يجيب على نفس الأسئلة إجابة صحيحة وذللك وفقاً للمعادلة

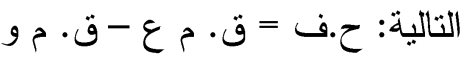

$$
\begin{aligned}
& \text { حيث: } \\
& \text { ح. ف = الاحتياج المعرفي. }
\end{aligned}
$$

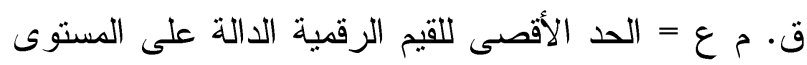
المعرفي المعياري المر اد تحقيقه.

ق. م و = القيمة الرقمية الدالة على المستوى المعرفي التيلية الو اقعي أي المحقق بالفعل.

المعارف التكنولوجية الزراعية الحديثة المتعلقة بإتتاج

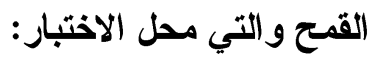

يقصد بها المعارف التكنولوجية المتعلقة بإنتاج القمح و التي يجب أن يكون الزراع ملمين بها، لكي يمكنهم زيادة

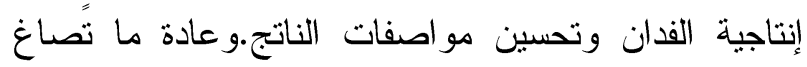
تلك المعارف الإرشادية الزراعية، على هيئة نوصيات،

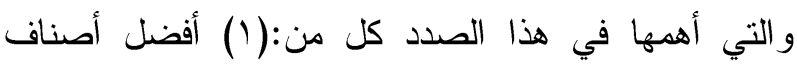

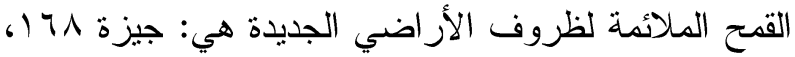

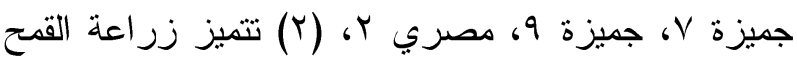
على مصاطب بكفاءة عالية في الحصول على محصول وفير وتحقيق العديد من المميزات، (r) استخدام السماد

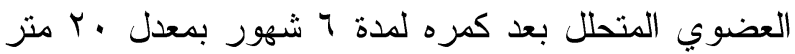

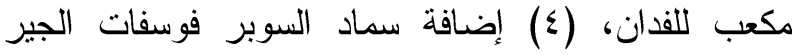
الأحادي بمعدل . . r كجم للفدان قبل الحرثة الثانية وعند

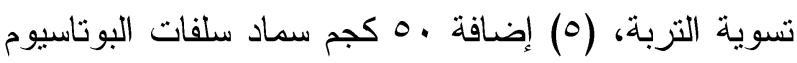
للفدان بعد شهر من الزراعة، (؟) إضافة الأمونيا السائلة

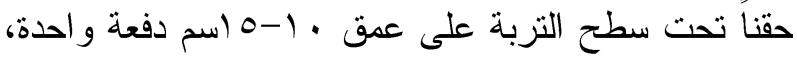


جدول ا. توزيع زراع القمح المبحوثين وفقاً لمستواهم المعرفي فيما يتعلق بتطبيق التوصيات التكنولوجية الزراعية

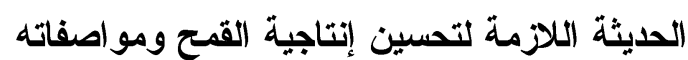

\begin{tabular}{|c|c|c|c|c|}
\hline$\%$ & الارجة" & $\%$ & العدد & فئات المستوى المعرفى (درجة) \\
\hline$T V, Y$ & $V .0$ & $\varepsilon V, \Gamma$ & $1 \leqslant 1$ & منخفض (•r-r (r) \\
\hline ro, & qщ. & r & $q \mu$ & 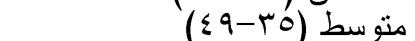 \\
\hline$r v,$. & 97. & $r_{1,0}$ & \rceil$\varepsilon$ & ررتفع (.0-0 (7)' \\
\hline $1 \ldots$ & ro90 & $1 \cdots$ & rqA & المجموع \\
\hline
\end{tabular}

* يقصد بها الدرجة الترجيحية القياسية(المعيارية) التي سبق ذكرها في المفاهيم الإجر ائية الواردة في هذا البحث .

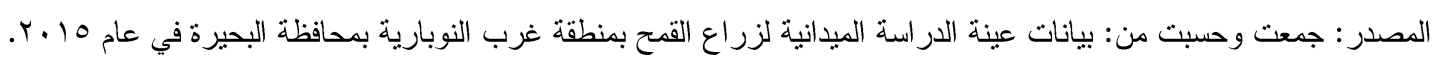

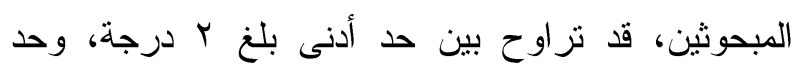

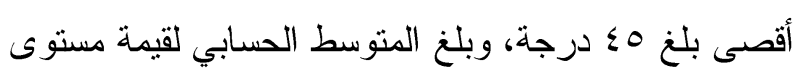

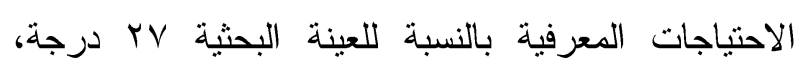

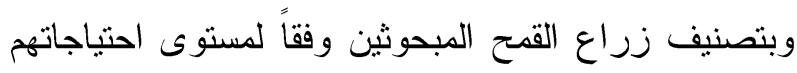
المعرفية فيما يتعلق بتطبيق التوصيات التكنولوجية

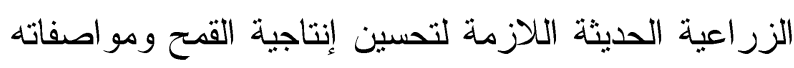
من خلال استخدام الدرجات الترجيحية القياسية(المعيارية) إلى ثلاث فئات، قد تبين أن فئة منخفضي الاحتياجات

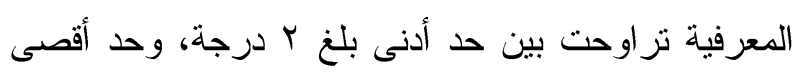

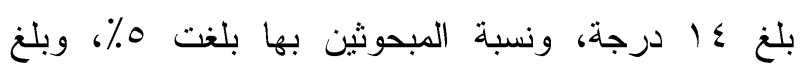
متوسط مستوى الاحتياجات المعرفية لتلك الفئة V درجات، في حين أن فئة متوسطي الاحتياجات المعرفية نر اوحت بين حد أدنى بلغ 10 درجة، وحد أقصى بلغ وب درجة، ونسبة ونية

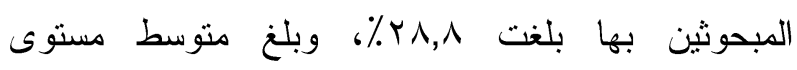
الاحتياجات المعرفية لتلك الفئة سب درجة، بينما فئة مرتفعي الاحتياجات المعرفية فقد تراوحت بين حد أدنى بلغ بلغ • ب درجة، وحد أقصى بلغ 0ـ درجة، ونسبة المبحوثين بها بلغت ب,77\%، وبلغ متوسط مستوى الاحتياجات المعرفية لتلك الفئة Vr درجة، أما بالنسبة لإجمالي زراع

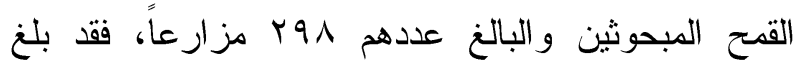
متوسط مستوى الاحتياجات المعرفية لهم VYدرجة، (جدول
ويتضح من بيانات الجدول(1) أن غالبية زراع القمح المبحوثين ذوى مستوى معرفي منخفض ومستوى متوسط، فيما يتعلق بنطبيق التوصيات التكنولوجية الزراعية الحديثة

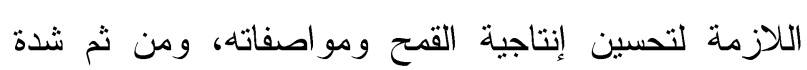
احتياجاتهم المعرفية المتعلقة بهذا الثأن. ويوضح الجدول(r) توزيع زراع القمح المبحوثين وفقاً لمستوى إجاباتهم على الأسئلة التي تقيس حصيلة معارفهم فيما يتعلق بتطبيق التوصيات التكنولوجية الزراعية الحديثة

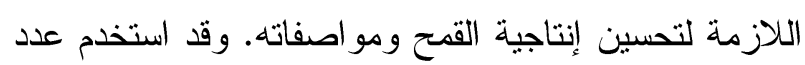
سا عبارة تحدد المستوى المعرفي وتبين أوجه القصور و إلى أي مدى يمكن تحسين المستوى المعرفي لهم. وتبين

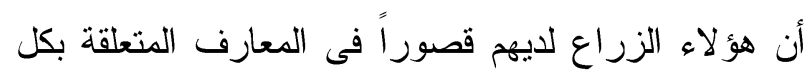

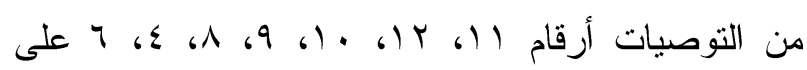

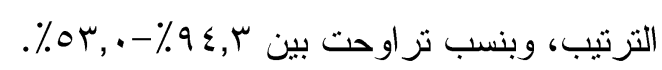

ويتبين من النتائج الواردة بالجدول(r) ارتفاع نسبة زراع القمح المبحوثين الذين لا يعرفون كل من التوصيات

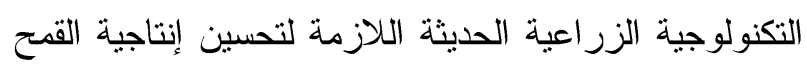

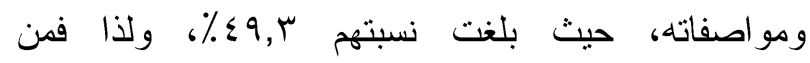
الضروري قيام الجهاز الإزشادي الزراعي بتخطيط وتتفيذ

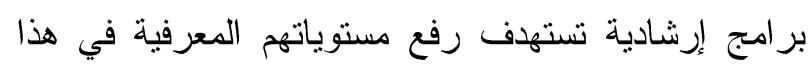

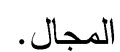
ثانياً: الاحتياجات المعرفية لزراع القمح المبحوثين: أوضحت النتائج البحثية أن المدى الفعلي للقيم الرقمية المعبرة عن مستوى الاحتياجات المعرفية لزراع القمح 
جدول r. توزيع زراع القمح المبحوثين"وفقاً لمدى معرفته بكل توصية على حده من التوصيات التكنولوجية الزراعية الحديثة اللازمة لتحسين إنتاجية القمح ومواصفاته

\begin{tabular}{|c|c|c|c|c|c|}
\hline \multicolumn{2}{|c|}{ لا بعرف } & \multicolumn{2}{|c|}{ بعزف } & \multirow[t]{2}{*}{ التوصية } & \multirow[t]{2}{*}{ r } \\
\hline$\%$ & 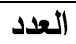 & $\%$ & العدد & & \\
\hline $\mid Y, 1$ & ry & $\Lambda \vee, 9$ & rYY & 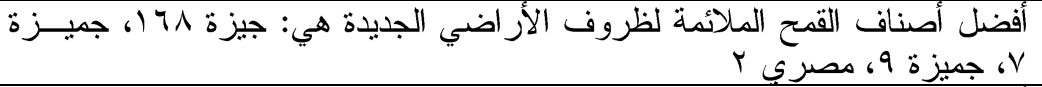 & 1 \\
\hline $1 \cdot, 1$ & r. & $\wedge 9,9$ & rYA & أنسب ميعاد لزر اعة القمح في القترة من 0 ا- ـ س نوفمبر & r \\
\hline$\varepsilon\rceil, r$ & $1 \mathrm{H}$ & or, $\mathrm{V}$ & 17. & أفضل الطرق لزر اعة القمح: الزر اعة العفيرباستعمال آلات التسطير & $r$ \\
\hline 70,1 & $19 \varepsilon$ & $\Gamma \varepsilon, q$ & $1 \cdot \varepsilon$ & معدل التقاوي المناسب في هذه الطريقة هو ـ كجم للفدان & $\varepsilon$ \\
\hline$r$ r & 79 & $\vee \neg, \wedge$ & rrq & العديد من الزر المميز اتلى مصاطب بكفاءة عالية في الحصول على محصول وفير وتحقيق & 0 \\
\hline or,. & 101 & $\varepsilon \vee$, & $1 \leq$ & 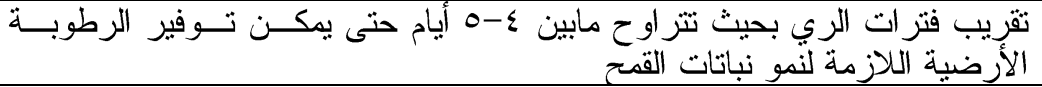 & 7 \\
\hline 19,1 & ov & $1 \cdot, 9$ & $r \leqslant 1$ & للفدان استخدام السماد العضوي المتحلل بعد كمره لمدة ج شهور بمعدل • ب متــر مكعــب & $\mathrm{V}$ \\
\hline 70,2 & 190 & $\Gamma \varepsilon, \uparrow$ & $1 \cdot r$ & 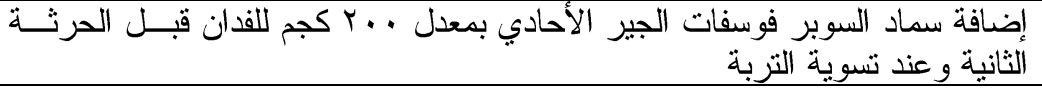 & $\Lambda$ \\
\hline $7 \wedge, 1$ & $r \cdot r$ & 1, 9 & 90 & إضافة ، 0 كجم سماد سلفات البوتاسيوم للفدان بعد شهر من الزر اعة & 9 \\
\hline$\vee \wedge, r$ & זru & 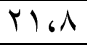 & 70 & إضافة الأمونيا السائلة حقنا تحت سطح التربة على عمق · 1-0 سم دفعة و احدة & 1. \\
\hline $9 \leqslant, r$ & YNI & $0, \mathrm{~V}$ & $1 \mathrm{~V}$ & فقد الأمونة محصول القمح بعد ب-ع أيام من الحقن مع عدم التأخير في الزر اعة لعــدم & 11 \\
\hline $94, r$ & rVo & $\mathrm{V}, \mathrm{V}$ & r & مكافحة الحشائش بالمبيدات في الأرض الموبو ءة قبل الحقن & Ir \\
\hline 17,1 & $\varepsilon \Lambda$ & $\Delta r, q$ & ro. & يبدأ الحصاد في أو ائل شهر مايو عند الصفر ار السلامية العليا التي تحمل السنبلة في & r \\
\hline$\varepsilon 9, r$ & $1 \leqslant V$ & $0 \cdot, \mathrm{V}$ & 101 & 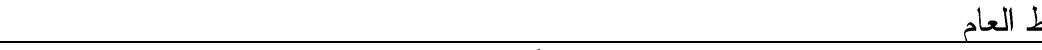 & 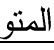 \\
\hline
\end{tabular}

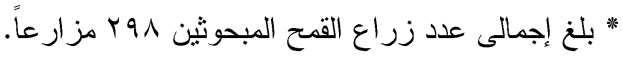

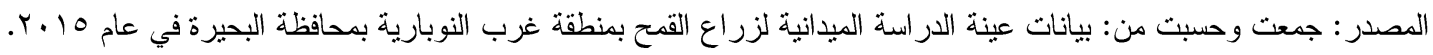

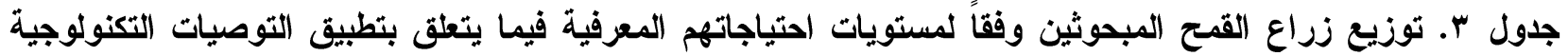
الزراعية الحديثة اللازمة لتحسين إنتاجية القمح ومواصفاته باستخدام الارجات الترجيحية القياسية

\begin{tabular}{|c|c|c|c|}
\hline متوسط مستوية للفئة الاحتياجات & عدد المبحوثين فى كل فئهة & مجموع درجات الفئة & فئات مستوى الإحتياجات \\
\hline V & $0 \leqslant$ & $\varepsilon \cdot Y$ & منخفضة (أقل من 0 1) \\
\hline r & $1 \cdot r$ & (r.A & متوسطة (0-10-r9) \\
\hline rV & $1 \leqslant Y$ & or91 & 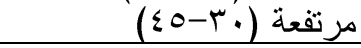 \\
\hline YV & rqA & $\wedge \cdots \wedge$ & المجمو ع \\
\hline
\end{tabular}

المصدر : جمعت وحسبت من: بيانات عينة الدر اسة الميدانية لزر اع القمح بمنطقة غرب النوبة النوبارية بمحافظة البحيرة في عام 10 ــr. بتضح من بيانات الجدول(r) أن غالبية زراع القمح الزراعي بتخطيط وتتفيذ برامج إرشادية تستهدف تتمية المبحوثين تقع في فئتي الاحتياجات المعرفية المرتفعة معارف زراع القمح وتحسين مهار اتهم وتغيير اتجاهاتهم، والمتوسطة فيما يتعلق بتطبيق التوصيات التكنولوجية حتى يتمكنوا من الاستفادة من التقنيات الحديثة التي تحقق الزر اعية الحديثة اللازمة لتحسين إنتاجية القمح ومواصفاته هذا الغرض، بما يكفل زيادة قدرتهم على الاستغلال الفعال (90\%)، وهذا الأمر يتطلب ضرورة قيام الجهاز الإرشادي 
a $=$ a عندما تكون قيمة المتغير المستقل xy

$$
\begin{aligned}
& \text { الأفقي صفر). } \\
& \text {. معامل انحدار = b }
\end{aligned}
$$

ويتضح من النتائج السابقة معنوية هذه الدالة، حيث

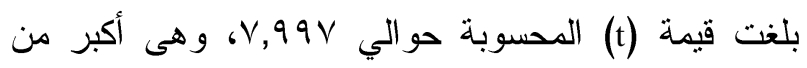

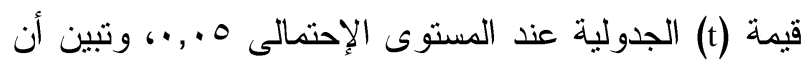

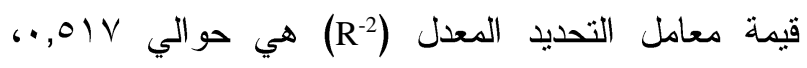
وبالتالي فإن متغير "الاستعداد للتغيير وتقبل الأفكار الجديدة" مسئول عن تقسير حوالي بr\% من جملة التغيير الممكن حدوثه في مستوى الاحتياجات المعرفية لزراع القمح من من

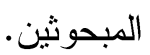

وتوضح قيمة "b" (معامل انحدار xy) أنه كلما زاد الاستعداد للتغيير وتقبل الأفكار الجديدة لدى المبحوثين بدرجة معيارية قياسية، يكون من المتوقع ارتفاع مستوى

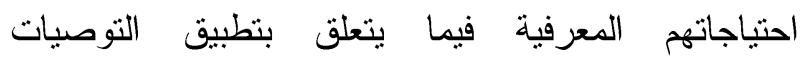

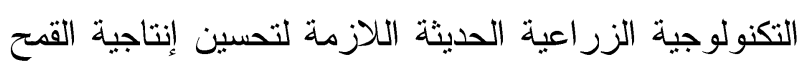
ومو اصفاته بحو الي ا , ا درجة ترجيحية قياسية. وتبين من التحليل الوصفي والإحصائي أن المدى الفعلي

للقيم الرقية المعبرة عن متغير "المساحة المنزرعة قمحاً"

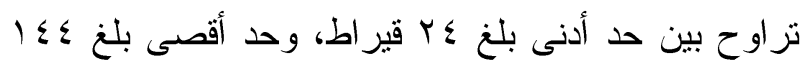

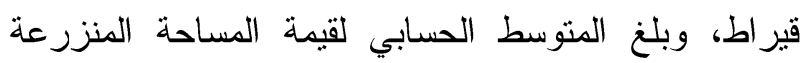
قمحاً بالنسبة للعينة البحثية بو قير اطن ومن خلال استخدام الدرجات الترجيحية القياسية تم توزيع المبحوثين إلى ثلاث

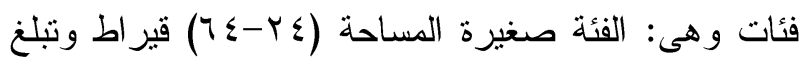
نسبتها Y, ٪٪، وبدرجة ترجيحية قياسية تبلغ ، وس درجة،

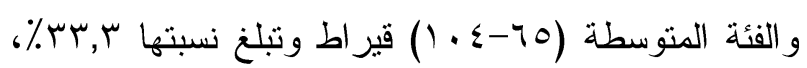
وبدرجة ترجيحية قياسية بلغت ؟و ؟ب درجة، أما الفئة

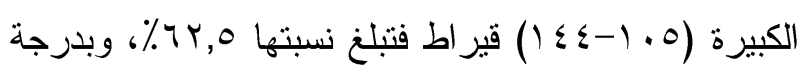

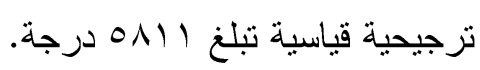

لما بحوزتهم من أر اضى منزرعة بمحصول القمح، وتحقيق أكبر صافى عائد للفدان، وبالتالي ارتفاع مستويات دخولهم. ثالثاً: أهم العوامل المؤثرة على مستوى الاحتياجات المعرفية لزراع القمح المبحوثين:

أوضح التحليل الوصفي والإحصائي لأهم المتغيرات

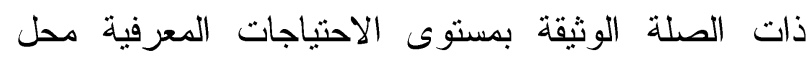
الاهنمام أن متغير "الاستعداد للتغيير وتقبل الأفكار الجديدة" تراوح بين حد أدنى بلغ درجة و احدة، وحد أقصى بلغ 19 درجة، وبلغت قيمة المتوسط الحسابي 11 درجة، وبتصنيف ولته المبحوثين وفقاً للارجات الترجيحية القياسية المعبرة عن هذا ونها المتغير إلى ثلاث فئات، فتبين أن الفئة المنخفضة الاستعداد (1-7) درجة، نسبتهر 0.9\%، وبدرجة ترجيحية قياسية تبلغ ع ع درجة، و المتوسط الاستعداد (Y-V I I) درجة، نسبتهم \%,0,

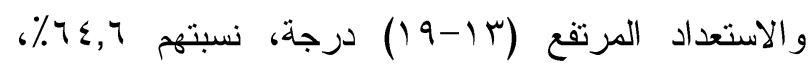
وبدرجة ترجيحية قياسية تبلغ r الو درجة.

وبإجراء التحليل الكمي والإحصائي في صورة دالة خطية أوالارجة الأولى تشتمل على كل من: منغير

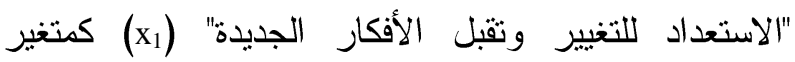
مستقل، ومتغير"مستوى الاحتياجات المعرفية" (y) كمتغير

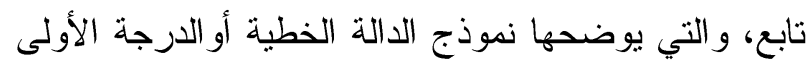
$\mathrm{y}=\mathrm{a} \pm \mathrm{b} \mathrm{x}_{1}$ $\mathrm{y}=38.969+1.079 \mathrm{x}_{1}$

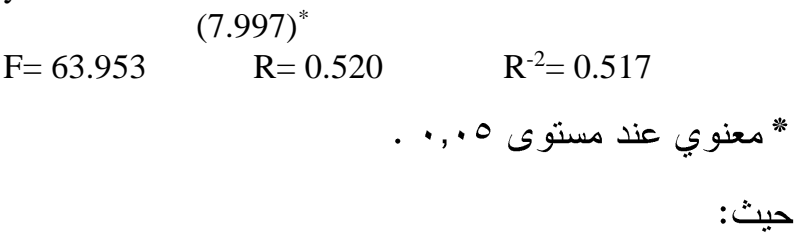
مستوى الاحتياجات المعرفية فيما بتعلق بتطبيق $=$ y التوصيات التكنولوجية الزر اعية الحديثة اللازمة لتحسين إنتاجية القمح ومو اصفاته (بالدرجة الترجيحية القياسية). الاستعداد للتغيير وتقبل الأفكار الجديدة(بالدرجة المعيارية القياسية). 
واتضح من التحليل الوصفي والإحصائي أن

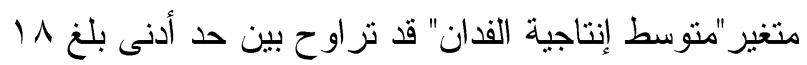
أردب، وحد أقصى بلغ rV أردب، وبلغت قيمة المتوسط

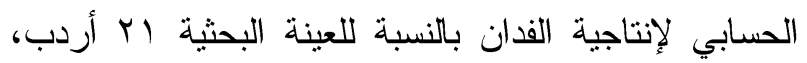
وتم توزيع المبحوثين وفقاً لمتوسط إنتاجية فدان القمح لديهم إلى ثلاث فئات من خلال استخدام الدرجات الترجيحية

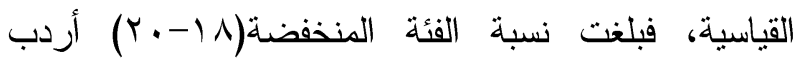

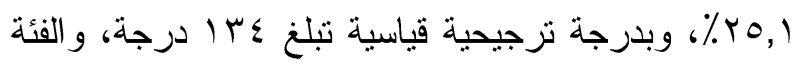
المتوسطة (IM-Y) أردب بلغت نسبتها وبدرجة ترجيحية قياسية بلغت عـا درجة، أما الفئة

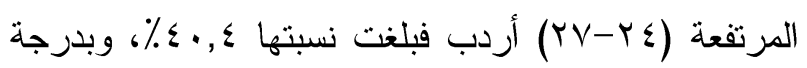
ترجيحية قياسية تبلغ 7 اب درجة. وقد تم إجر اء التحليل الكمي و الإحصائي في صورة دالة خطية أب الدرجة الأولى تتطوي على كل من: متغير

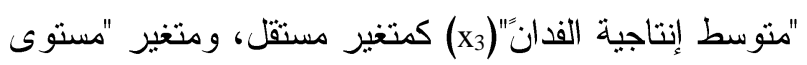
الاحتياجات المعرفية" (y) كمتغير تابع، والتي يوضحها نموذج الدالة الخطية (الدرجة الأولى) الآتية: $\mathrm{y}=\mathrm{a} \pm \mathrm{bx}_{3}$

$\mathrm{y}=100.654-3.432 \mathrm{x}_{3}$

$(-21.398)^{* * *}$

$\mathrm{F}=457.876 \quad \mathrm{R}=0.607$

$$
\mathrm{R}^{-2}=0.606
$$

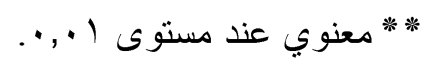$$
\text { حيث: }
$$

مستوى الاحتياجات المعرفية فيما يتعلق بتطبيق = y التوصيات التكنولوجية الزر اعية الحديثة اللازمة لتحسين إنتاجية القمح ومو اصفاته (بالدرجة الترجيحية القياسية).

$$
\text { =x3 = متوسط إنتاجية الفدان (بالأردب). }
$$

= a عندما نكون قيمة المتغير المستقل x3 على المحور y

$$
\text { (الأفقي صفر). }
$$$$
\text { . معامل انحدار = b }
$$

ونم إجراء التحليل الكمي والإحصائي في صورة دالة خطية أب الدرجة الأولى نشتمل على كل من: متغير "المساحة المنزرعة قمحاً" (x2) كمتغير مستقل، ومتغير "مستوى الاحتياجات المعرفية" (y) كمتغير تابع، و التي يوضحها نموذج الدالة الخطية (الدرجة الأولى) الآتية: $\mathrm{y}=\mathrm{a} \pm \mathrm{b} \mathrm{x}_{2}$ $y=3.026+0.593 x_{2}$

$$
\begin{aligned}
& \mathrm{F}=395.638 \quad \mathrm{R}=0.715 \quad \mathrm{R}^{-2}=0.714 \\
& \text { * معنوى عند مستوى ا +, "•. } \\
& \text { حيث: }
\end{aligned}
$$

= y التوصيات التكنولوجية الزر اعية الحديثة اللازمة لتحسين إنتاجية القمح ومو اصفاته (بالدرجة الترجيحية القياسية). =x2 = a عندما تكون قيمة المتغير المستقل x2 على المحور

$$
\begin{aligned}
& \text { الأفقي صفر). } \\
& \text {. معامل انحدار = b }
\end{aligned}
$$

وتبين أن تلك الدالة معنوية عند المستوى الإحتمالى

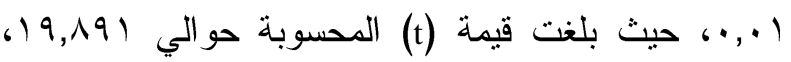
وهى أكبر من قيمة (t) الجدولية، كما تبين أن قيمة معامل

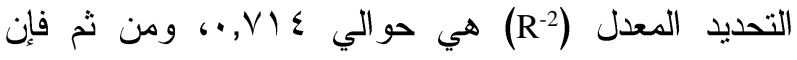
متغير "المساحة المنزرعة قمحاً" مسئول عن تفسير حوالي

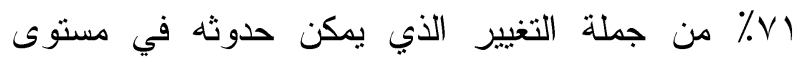
الاحتياجات المعرفية لزراع القمح المبحوثين. وتوضح قيمة "b" (معامل انحدار x2) أنه كلما زادت المساحة المنزرعة قماً لدى المبحوثين بقير اط واحد، فمن المتوقع ارتفاع مستوى احتياجاتهم المعرفية فيما يتعلق بتطبيق التوصيات التكنولوجية الزراعية الحديثة اللازمة لتحسين إنتاجية القمح ومواصفاته بحوالي 09, • درجة تزجيحية قياسية. 
مستوى الإحتياجات المعرفية فيما يتعلق بتطبيق y التوصيات التكنولوجية الزر اعية الحديثة اللازمة لتحسين إنتاجية القمح ومو اصفاته (بالدرجة الترجيحية القياسية). = x

\section{المعيارية القياسية).}

=x2 = x3

وتبين أن تلك الدالة معنوية عند المستوى الإحتمالى

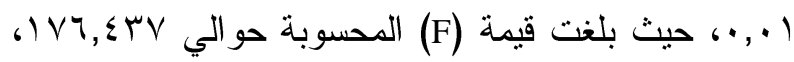
وهى أكبر من قيمة (F) الجدولية، كما تبين أن قيمة معامل

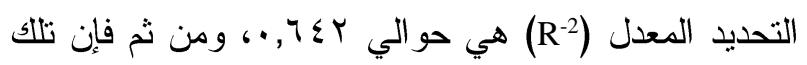
المتغيرات الثلاثة المستقلة التي تضمنها هذا النموذج عان مجتمعة مسئولة عن تفسير حوالي ؟ ؟٪ من جملة التغيير الذي يمكن حدوثه في مستوى الاحتياجات المعرفية للزراع المبحوثين فيما يتعلق بتطبيق التوصيات التكنولوجية الزر اعية الحديثة اللازمة لتحسين إنتاجية القمح ومو اصفاته، بنطين ويعنى ذللك أن هنالك متغيرات أخرى خارج هذا النموذج المستخدم فئ التحليل ذات تأثير على مستوى الاحتياجات

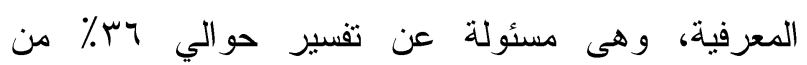
التغييرات التي تطر أ على مستوى الاحتياجات المعرفية. وتوضح قيم معاملات المرونة الجزئية للمتغيرات الداخلة في الدالة السابقة أنه كلما ارتفع مستوى الاستعداد

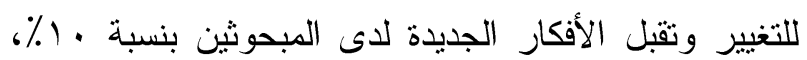
يكون من المتوقع ارتفاع مستوى احتياجاتهم المعرفية بنسبة

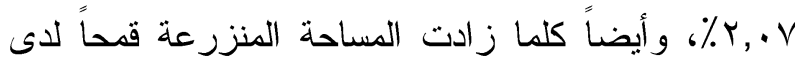

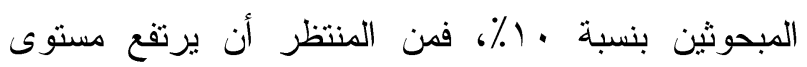

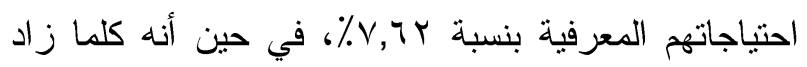

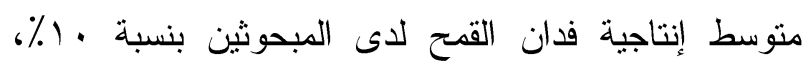
يكون من المتوقع انخفاض مستوى احتياجاتهم المعرفية
و إتضح من النتائج السابقة معنوية هذه الدالة عند المستوى الإحتمالى 1 +, •، حيث بلغت قيمة (t) المحسوبة

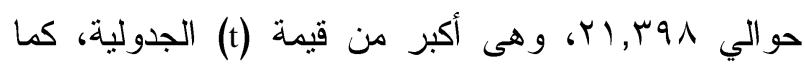

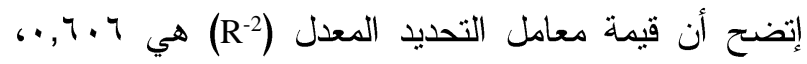

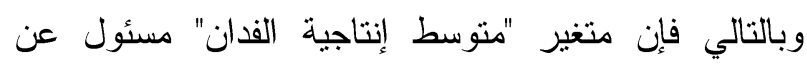
تفسير حوالي الج٪ من جملة التغيير الذي يمكن حدوثه في مستوى الاحتياجات المعرفية لزر اع القمح المبحوثين. وتثير قيمة "b" (معامل انحدار x3) إلى أنه كلما زادت إنتاجية فدان القمح لدى المبحوثين بحوالي أردب واحد، يكون من المتوقع انخفاض مستوى احتياجاتهم المعرفية فيما يتعلق بتطبيق التوصيات التكنولوجية الزراعية الحديثة

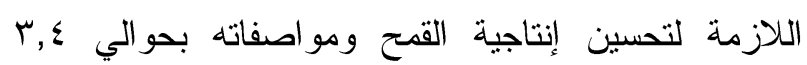

درجة تزجيحية قياسية.

ولمعرفة درجة تأثير كل من المتغيرات المستقلة الثلاث

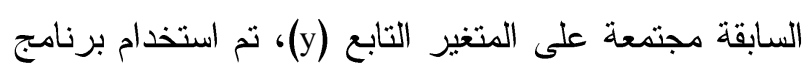
(SPSS) التي تعبر معاملات انحدارها عن المرونة الجزئية، أبي درجة التأثير النسبي لكل متغير من المتغيرات المستقلة على هلى المتغير التابع، ومن ثم التوصل إلى التغيرات التي تطرأ على مستوى الاحتياجات المعرفية للمبحوثين، و التي تمثل نتائجها أسس استخلاص التوصيات التي تستهدف سد الفجوة المعرفية لاى المبحوثين، وكذلك التغلب على المشكلات التي تواجههم في مرحلتي الإنتاج و التسويق لمحصول وحلى القمح، وانطوت هذه الدالة على مستوى الإحتياجات المعرفية كمتغير تابع (y)، وثناثثة متغيرات مستقلة والتي هده

يوضحها نموذج الدالة اللوغاريتمية المزدوجة الآتية: $\operatorname{Ln} y=\operatorname{Ln} b_{0}+b_{1} \operatorname{Ln} x_{1}+b_{2} \operatorname{Ln} x_{2}+\ldots+b_{n} \operatorname{Ln} x_{n}$ $\operatorname{Ln} \mathrm{y}=\operatorname{Ln} 5.750+0.207 \operatorname{Ln} \mathrm{x}_{1}+0.762 \operatorname{Ln} \mathrm{x}_{2}-1.919 \operatorname{Ln} \mathrm{x}_{3}$

$\mathrm{F}=176.437 \quad \mathrm{R}=0.645 \quad \mathrm{R}^{-2}=0.642$

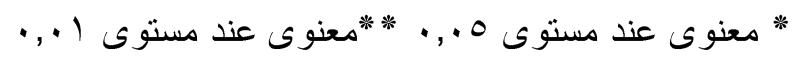


و الذي يتضح منه أن مشكلة "انخفاض سعر محصول القمح وتذبذب سعره" قد تصدرت قائمة المشكلات، وكانت الأهمية

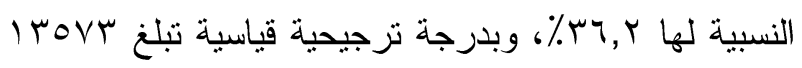
درجة، وجاءت مشكلة "ارتفاع أسعار مستلزمات الإنتاج وندرة المياه" في درجة الأهمية النسبية الثانية وتبلغ

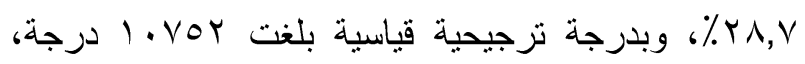

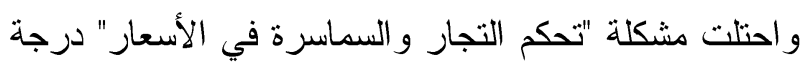

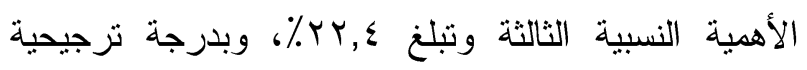

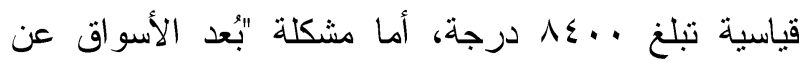

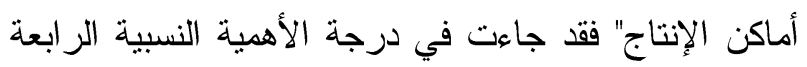

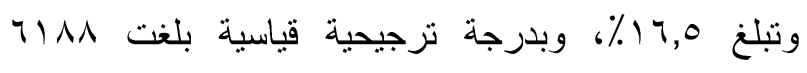
درجة، بينما جاءت مشكلة "ارتفاع أجور العمالة الزراعية وندرتها وقلة إنتاجيتها" في درجة الأهمية النسبية الخامسة

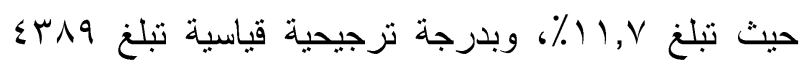
درجة، واحتلت مشكلة "ارتفاع تكلفة وعدم تو افر وسائل

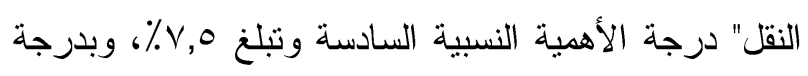

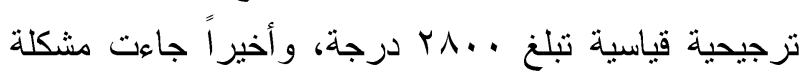
"قصور وضعف جهاز الإرشاد الزر اعي" في درجة الأهمية النسبية السابعة وتبلغ ج, ؟\%، وبدرجة نرجيحية قياسية بلغت

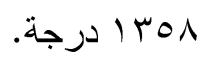

وتؤكد قيمة المرونة الكلية(-90,9.) وهي تساوى مجموع المرونات الجزئية للمتغيرات المعنوية إحصائياً. آخذاً في الاعتبار الإشارات الجبرية. وهنا الإشارة سالبة وتؤكد العلاقة العكسية على أن حُسن تخطيط وتتفيذ البرنامج الإرشادي المتعلق برفع المستوى المعرفي للزراع بتطبيق التوصيات التكنولوجية الزراعية الحديثة اللازمة لتحسين إنتاجية القمح ومواصفاته، سوف يساعد ذلك في خفض مستوى احتياجاتهم المعرفية الخاصة بهذا الثأن

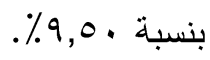
وجدير بالذكر أنه نم عمل نركيبات أخرى، كل منها

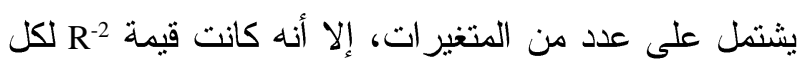

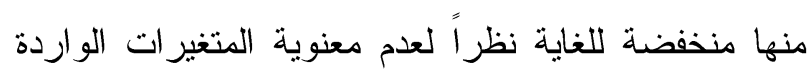

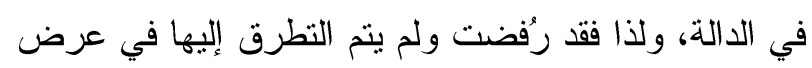
النتائج.

رابعاً: الأهمية النسبية للمشكلات التي تواجه زراع القمح المبحوثين:

يواجه زراع القمح المبحوثين العديد من المشكلات الإنتاجية و التسويقية التي تحول دون زيادة مستوى دخولهم،

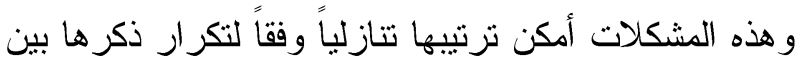
زراع القمح المبحوثين على النحو المبين في الجدول(ع)، جدول ع. الأهمية النسبية للمشكلات التي تواجه زراع القمح المبحوثين باستخدام الارجات الترجيحية القياسية بمنطقة

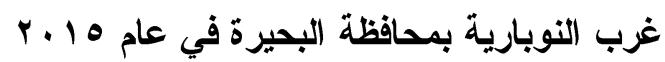

\begin{tabular}{|c|c|c|c|c|}
\hline 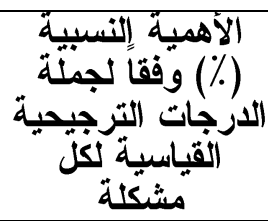 & 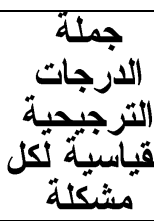 & 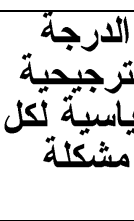 & 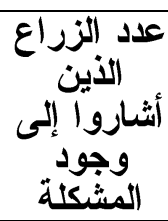 & 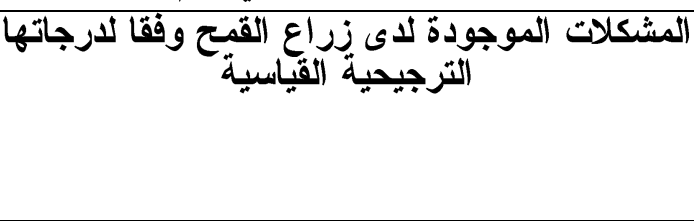 \\
\hline r Y,Y & IMOVT & $\leqslant 9$ & TVV & إنخفاض سعر محصول القمح وتذبذب سعره \\
\hline$r \wedge, \vee$ & 1. Vor & $\leqslant r$ & rot & إرتفاع أسعار مستلزمات الإنتاج وندرة المياه \\
\hline 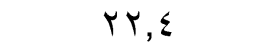 & $\Lambda \varepsilon \ldots$ & ס r & $r \varepsilon$. & تحكم التجار و السماسر \\
\hline 17,0 & $7) \wedge \Lambda$ & rᄉ & YYI & عن أماكن ا \\
\hline $11, \mathrm{~V}$ & $\leqslant \mu \wedge q$ & r) & $r \cdot q$ & إزرتفاع أجور العمالة الزر أعيةٌ وندرتها \\
\hline$V, 0$ & r^.. & $1 \varepsilon$ & r.. & إرتفاع تكلفة و عدم تو افر وسائل النقل \\
\hline$r, 7$ & Iron & V & $19 \leq$ & قصور وضعف جهاز الإرشاد الزر اعى \\
\hline
\end{tabular}

المصدر : جمعت وحسبت من: بيانات عينة الدراسة الميدانية لزراع القمح بمنطقة غرب النوبارية بمحافظة البحيرة فى عام 10 . r. 
شادية حسن فتحي، ع19 1 (: تخطيط البر امج الإرشادية الزر اعية،

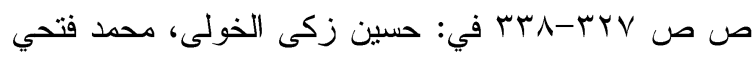

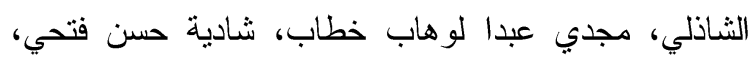
الإرشاد الزر اعي، كتاب الصقر، الإسكندرية.

عبد السلام أحمد جمعة، هـ ـ. التجربة المصرية في تتمية

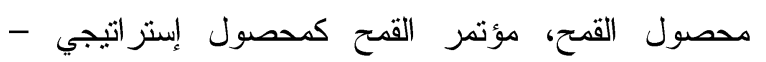

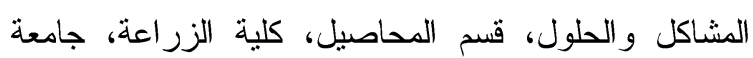
الإسكندرية.

عبد العاطى حميدة محمد سكر، 997 (: در اسة اتجاهات الزراع

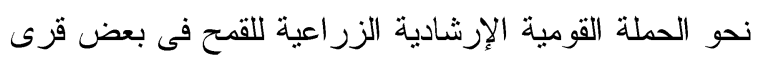

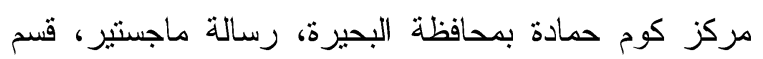
الإزشاد الزر اعي، كلية الزر اعة، جامعة الإسكندرية . مجدي الشوربجى، ع991: الاقتصاد القياسي (النظرية و التطبيق)، الدار المصرية اللبنانية، القاهرة، الطبعة الأولى. محمود عبد السلام حسين النحاس، 11 ـ ץ: الاستفادة من النظام الخبير للقمح بالمر اكز الإرشادية الزر اعية بمحافظة البحيرة،

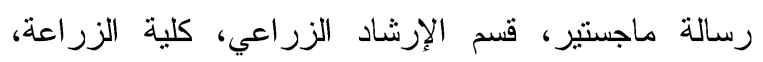
جامعة الإسكندرية. محمد مصطفى شييه، صالح نصار النصار، 9AV Iا: تقدير الاحتياجات التدريبية لمزارعي القمح بمنطقة القصيم، كلية

الزر اعة، جامعة الملك سعود، المملكة العربية السعودية. مديرية الزراعة بالنوبارية، ع ا.ب؟: سجلات قسم الإحصاء، بيانات غير منشورة.

وزارة الزراعة واستصلاح الأراضي، ل1 لـr: أهم التوصيات

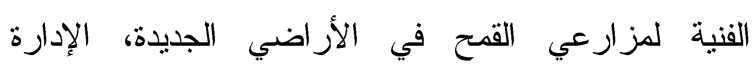
المركزية للإزشاد الزر اعي، مركز الدعم الإعلامي بمريوط.

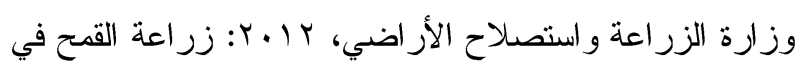

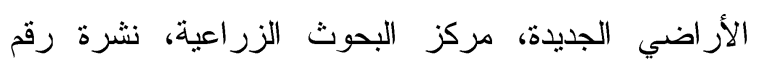

\section{التوصيات}

ا - نظر اً لما أوضحته النتائج البحثية من وجود قصور لدى زراع القمح في المعارف المتعلقة ببعض التوصيات التكنولوجية الزراعية الحديثة اللازمة لتحسين إنتاجية القمح ومو اصفاته، فمن الضروري عمل دورات تدريبية وتتفيذ برامج إرشادية تستهدف رفع مستو اهم المعرفي فيما يتعلق بتلك التوصيات، وخاصة المرتبطة منها بكل

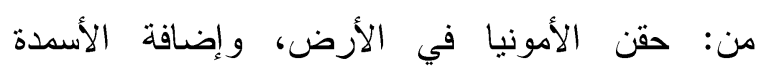
الكيماوية، وكمية الثقاوي المناسبة للفدان، ومعدلات الري اللازمة لنمو نباتات القمح. r- التوسع في إنشاء الصوامع المتطورة، التي تلزم لتخزين القمح بشكل آمن يحافظ على كمية وجودة المحصول من النساء الفقد أو التلف. بكن. بـ تعاقد الحكومة مع الزراع لثراء محصول القمح منهم ودعم سعره. ع - تدخل الحكومة لتوفير مستلزمات الإنتاج في الجمعيات التعاونية الزر اعية بأسعار منخفضة. 0ـ تتظيم الري وزيادة كميات المياه المتاحة لزر اع القمح. 7- إنشاء جمعيات تسويقية و إقامة أسواق في أماكن إنتاج القمح.

V- توفير الميكنة الزر اعية نظر اً لندرة العمالة وقلة إنتاجيتها وارتفاع أجور ها.

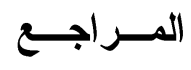

$$
\begin{aligned}
& \text { أحمد محمد عمر، بلو ا: المرجع في الإرشاد الزراعي، دار } \\
& \text { النهضة العربية، القاهرة. }
\end{aligned}
$$

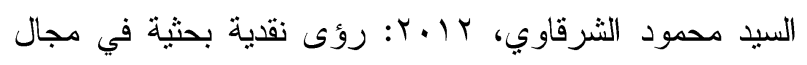

$$
\begin{aligned}
& \text { العلوم الاقتصادية والاقتصادية الزراعية فيما بين النظرية } \\
& \text { و التطبيق، أبو الخير للطباعة، الإسكندرية، الجزء الثاني. }
\end{aligned}
$$




\title{
ABSTRACT \\ Range Needs of Wheat Farmers to the New Agricultural Knowledge Technology Concerning Improving the Productivity and Specifications of Wheat in West Nubaria Area in El-Beheira Governorate
}

\author{
Ahmed M. A. Ghozlan
}

This research is mainly to determine Range Needs of Wheat Farmers to the New Agricultural Knowledge technology Concerning Improving the Productivity and Specifications of Wheat, and then determine to what range can help agricultural extension devices to raise the level of cognitive in this regard, as well as to determine the relative importance of the problems hinder wheat farmers increase their knowledge on the subject of interest. It was necessary, using survey data collection by personal interview of research random sample of wheat farmers in the villages of Imam Malik, and friend Joseph West Nubaria area Beheira number of vocabulary 298 respondents representing $24 \%$ of the overall research.

It is analytical and descriptive statistical level of knowledge needs to study wheat farmers regarding the application of modern agricultural technology recommendations for improving the productivity of wheat and specifications, has learned a large number of research results that have been invoked in the recommendations and the most important are:

1- Show through the using of standard grades measure the level of knowledge that the majority of wheat farmers surveyed people with cognitive level is low and the level of the average rate of about $63.0 \%$, with respect to the application of agro-technological recommendations modern needed to improve wheat and specifications productivity, and thus the severity of cognitive their needs related to in this regard.

2- Show the high proportion of wheat farmers of respondents who do not know all of the technological recommendations agricultural modern needed to improve wheat productivity and specifications, they have accounted for about $49.3 \%$, as well as having deficiencies in knowledge on a number of recommendations to the farmers who have a good knowledge of those recommendations in the study, and who do not exceed their percentage only about $50.7 \%$ of the total respondents wheat farmers.
3- Show through the using of standard shoot-grading of the need for cognitive, that the highest value reflects the level Needing cognitive wheat farmers respondents regarding the application of agrotechnological recommendations modern needed to improve wheat productivity and specifications is 45 degrees, and the minimum value is zero degrees, was the distribution of the respondents according to the levels their need Cognitive into three classes: Class Needing low cognitive, accounting for about 5\%, and middle-class Needing cognitive, accounting for about $28.8 \%$, and finally the category Mrtfie Needing cognitive, accounting for about $66.2 \%$.

4- In light of the results of statistical analysis and quantitative it turned out to be the most important variables influencing the level of knowledge needs to respondents regarding the application of agrotechnological recommendations modern needed to improve wheat productivity and specifications are: willingness to change and accept new ideas, and the acreage under wheat, the average acre productivity. And then these variables should be of interest and the focus of agricultural extension programs, which directs toward wheat farmers and aimed at raising their level of cognitive regard to the recommendations in the study work.

Show through the using of standard shoot-grades that the most important problems that hamper the wheat farmers Almibhothin increase their knowledge regarding the application of agro-technological recommendations modern needed to improve wheat productivity and specifications according to their answers on the overall standings countdown follows: low price of wheat and the volatility of its price, high production and water scarcity input prices Traders, brokers and control in prices, after the market for the production places, high agricultural labor and scarcity wages and lack of productivity, high cost and the lack of means of transport, and Qsourodaf agricultural extension device, which ranged from the relative importance of grades shootout between $36.2 \%$ to $3.6 \%$ ). 